Canadian University Music Review

Revue de musique des universités canadiennes

\title{
Matériau et composition
}

Sur trois ouvres vocales de Luciano Berio

\section{Philippe Albèra}

Numéro 4, 1983

URI : https://id.erudit.org/iderudit/1013898ar

DOI : https://doi.org/10.7202/1013898ar

Aller au sommaire du numéro

\section{Éditeur(s)}

Canadian University Music Society / Société de musique des universités canadiennes

\section{ISSN}

0710-0353 (imprimé)

2291-2436 (numérique)

Découvrir la revue

Citer cet article

Albèra, P. (1983). Matériau et composition : sur trois œuvres vocales de Luciano Berio. Canadian University Music Review / Revue de musique des universités canadiennes, (4), 66-94. https://doi.org/10.7202/1013898ar

(c) Canadian University Music Society / Société de musique des universités canadiennes, 1983
Ce document est protégé par la loi sur le droit d'auteur. L'utilisation des services d'Érudit (y compris la reproduction) est assujettie à sa politique d'utilisation que vous pouvez consulter en ligne.

https://apropos.erudit.org/fr/usagers/politique-dutilisation/ 


\title{
MATÉRIAU ET COMPOSITION Sur trois ouvres vocales de Luciano Berio
}

\author{
Philippe Albèra
}

En 1956, Luciano Berio, Umberto Eco et Furio Colombo décidèrent "d'écrire une représentation, Opera Aperta, sur le naufrage du Titanic ". ${ }^{1}$ Cette date signale, au niveau des projets, le début des préoccupations du compositeur italien pour les rapports entre texte, action théâtrale et musique, dans le contexte des travaux de l'avant-garde italienne qui marque la fin des années cinquante et le début des années soixante. ${ }^{2}$ Mais Opera, aboutissement transformé de ce projet, réalisé par Berio seul, ne vit le jour qu'en 1970. Et la série des œuvres vocales très importantes que Berio composa dans les années soixante est introduite par une œuvre électro-acoustique : Tema (Omaggio a Joyce), réalisée en 1958. Il est nécessaire, avant d'aborder plus en détail certaines de ces ouvres, de rappeler brièvement le contexte dans lequel elles s'inscrivent. À la fin des années cinquante, en effet, la " crise " de la musique sérielle engendre un éclatement stylistique sans précédent : des compositeurs comme Stockhausen, Boulez, Berio, Pousseur, etc, assouplissent leur technique d'écriture et cherchent à réaliser des formes ouvertes, mobiles, évolutives : Stockhausen dans Klavierstück IX (1956) ou Zyklus (1959), Boulez dans la 3ème Sonate pour piano (1955-57) ou les Structures II (1956-61), Pousseur avec Mobile (1956-58) et Répons (1960), Berio avec Sequenza I (1958) et Tempi Concertanti (1959). Cette investigation d'une nouvelle conception de la forme musciale (et par conséquent de toutes les dimensions de l'œuvre, de la communication même) trouve très rapidement sa formulation théorique grâce à Umberto Eco, dans un ouvrage qui reprend significativement le titre projeté pour la représentation imaginée avec Berio: Opera Aperta (1962), mais le livre tire son origine d'une conférence prononcée 
sur ce thème par Eco en 1958. Le livre d'Eco cite, au début, certaines œuvres musicales mentionnées ci-dessus, et consacre la moitié de son travail à l'analyse de l'œuvre de Joyce, qui a exercé sur Berio (1959) et sur Boulez (1964) une fascination stimulante.

Il faut encore noter que cette " rupture " avec le sérialisme sévère du début des années cinquante s'accompagne d'une investigation importante des moyens électro-acoustiques : Gesang der Jünglinge (1955-56) et Kontakte (1959-60) de Stockhausen, Tema (1958), Différences (1958-59) et Visage (1961) de Berio, Scambi (1957) de Pousseur, Poésie pour pouvoir (1958) de Boulez. Certaines de ces œuvres mêlent l'électronique aux instruments traditionnels. Elles ne sont certainement pas étrangères à la remise en question des principes édifiés auparavant, mais ce sujet dépasse le cadre de cet article.

Enfin, notons à la même époque une préoccupation nouvelle pour le théâtre musical et pour l'élaboration de nouveaux rapports entre langue et musique. Votre Faust (1960-67) de Pousseur, Originale (1961, à partir de Kontakte) de Stockhausen, Pli selon Pli (1957-62) de Boulez, et évidemment, les très nombreuses œuvres de Berio: Circles (1960), Passaggio (1962), Laborintus II (1965), Sequenza III (1965-66) et Opera (1970). C'est aussi l'époque où Kagel, qui se démarque clairement du mouvement sériel (cf. Kagel 1966 : 42), aborde le théâtre musical et instrumental. Dernier élément à signaler, le travail sur la voix, que Berio entend "libérer »: «... après Monteverdi, c'est toujours le modèle instrumental qui a prévalu. . . . Le parlé, c'est le bruit, le son sauvage qu'il n'est pas possible de noter. Le chanté, c'est le son noble... » ${ }^{3}$ En suivant l'exemple de Cage - «Utiliser le langage (en attendant quelque chose d'autre que la syntaxe) comme s'il était une source de sons qui peut être transformée en charabia $»^{3}$ - et en approfondissant les œuvres d'écrivains particulièrement préoccupés par la dimension sonore du langage (Joyce, Cummings, Sanguinetti ....), Berio a inventé, avec l'aide de Cathy Berberian, une «nouvelle vocalité ». La " nouvelle vocalité », dit Cathy Berberian, "c'est l'emploi des différents styles, des différentes émissions de la voix, l'utilisation du chant, de la parole, du bégaiement, de la toux, des rires, des pleurs, des gémissements, etc. ${ }^{4}$ Berio dit aussi : "Je tente de délivrer la voix de ce qui la limitait, faire qu'il n'y ait plus de frontières entre le parlé et le chanté. La composition pour voix devient la structure de "significations articulées ". " 5 
Cette transgression de l'espace musical traditionnel est ce qui a modifié en profondeur la pensée musicale. L'émancipation de la dissonance, l'usage d'accords complexes, libérés des fonctions harmoniques, l'introduction d'instruments à hauteurs non déterminées, ou de modes de jeu nouveaux, l'exploration et l'utilisation de moyens électro-acoustiques ont, depuis le début du siècle, considérablement enrichi et transformé le matériau musical. Ils ont du même coup remis en question les anciennes catégories de pensée, sans toutefois qu'une nouvelle " théorie » acceptée par tous s'impose, objectivant ce matériau à travers certains principes d'écriture. Le sérialisme a, pour une part, poursuivi cette utopie, à partir de la structuration des hauteurs (et accessoirement des autres paramètres). La génération de Darmstadt a tenté de généraliser ce mode de structuration aux durées, aux timbres et aux intensités. Néanmoins, la série est apparue avant tout comme un moyen d'organisation rationelle s'appliquant de façon privilégiée à des données que l'on peut convertir en nombres. Le timbre et les intensités, catégories phénoménales difficiles à hiéarchiser et n'ayant pas d'échelle absolue, ont offert une résistance particulière au traitement sériel, ainsi que l'ont remarqué assez vite certains compositeurs et quelques commentateurs. Pourtant, timbres et intensités étaient apparus, dés le début du siècle, comme des unités distinctives primordiales. Ceci tout particulièrement dans une période où le paramètre des hauteurs était laissé à une organisation très subjective, presque anarchique. Or en musique, les unités distinctives sont des unités de sens. Ce qui était jusqu'alors élément secondaire devenait élément structurel.

Toute une part du matériau nouveau dégagé par le XXème siècle était un matériau autrefois confiné dans le domaine du bruit, de l'indéterminé, un matériau exclu de l'espace musical. Les sons complexes (sons électroniques, clusters, ou accords) inanalysables auditivement en termes de hauteurs tempérées, doivent être différenciés (et devenir signifiants) au niveau de la densité, du timbre, des intensités, voire du geste. Cela implique de nouvelles catégories compositionelles, capables d'articuler ce matériau nouveau, de lier l'organisation de la micro-structure à une conception satisfaisante de la forme. Suivant ce que dit Adorno,

il reste nécessaire pour l'articulation de la musique de disposer de catégories formelles, même complètement 
modifiées, si on ne veut pas se contenter d'accumuler des sons. Il ne s'agit pas de restaurer les anciennes catégories, mais d'en forger d'équivalentes qui correspondent au nouveau matériau (1982 : 303).

Comment définir cette notion de matériau musical? Nous empruntons à Adorno :

Le matériau ne peut être conçu que comme ce avec quoi le compositeur opère et travaille. En ce sens, il n'est rien moins que, objectivé et réfléchi de façon critique, l'état des forces de production techniques auxquels les compositeurs sont confrontés à une époque donnée. Aspects physiques et historiques s'y chevauchent (ibid. : 302).

La question qui demeure est: quelles structures compositionnelles sont impliquées par le matériau? Berio, qui n'a jamais fétichisé la série, s'est rapidement éloigné d'une conception dominée par le paramètre des hauteurs. Il a cherché, à travers l'expérience du Studio di Fonologia Musicale de Milan, puis de la série des œuvres vocales durant les années soixante, de développer des modes de structuration propres à un matériau considérablement élargi. Boulez avait posé de la façon suivante ce problème du rapport entre matériau et structure :

Maintenant que nous avons un organisme comme la série dont la hiérarchie n'est plus fondée sur le principe d'identité par transposition mais au contraire sur des déductions localisées et variables, le bruit s'intègre plus logiquement à une construction formelle, à condition que les structures qui en sont responsables se fondent sur ses critères propres. Ils ne sont pas fondamentalement différents - acoustiquement parlant - des critères d'un son (1963: 43).

Et plus loin : "Au-delà de l'idée de mélange des éléments, encore naïve, se situe une dialectique structure-matériau selon laquelle l'une est le révélateur de l'autre " (ibid. : 45). Mais il n'y a pas de critères absolus applicables à un ensemble de situations : chaque œuvre réclame sa propre "méthode ", précisément en rapport avec la nature du matériau choisi.

C'est à partir de trois œuvres vocales utilisant des effectifs différents que je voudrais approfondir cette question de la relation entre matériau et composition. Ces trois œuvres de Berio 
datent des années soixante : il s'agit de Circles pour voix, harpe et percussions, Sequenza III pour voix seule, et $O \mathrm{King}$ pour voix, flûte, clarinette, violon, violoncelle et piano.

Circles $^{6}$ est composé à partir de trois textes du poète américain e.e. cummings : stinging, riverly, $n(0) w$. La voix est entourée d'une harpe et de deux groupes d'instruments à percussions, parmi lesquels beaucoup sont à hauteur indéterminée. Le choix de cet effectif indique d'emblée l'importance qu'auront des paramètres comme le timbre ou les intensités. Dans Circles, ils sont liés à la dimension phonétique des textes de cummings. Le poète a cherché une interpénétration de toutes les dimensions de l'écriture : phonétique, syntaxique, sémantique, gestuelle. . . Ces différentes dimensions apparaissent tantôt subordonnées à une présentation traditionnelle (comme dans "stinging "), elles opèrent alors de façon sous-jacente, tantôt l'aspect graphique les manifeste (comme dans " $\mathrm{n}(\mathrm{o}) \mathrm{w} »)$. L'organisation complexe de ces différents niveaux de l'œuvre débouche sur une multiplicité de lectures possibles qui a fasciné beaucoup de compositeurs contemporains, et qui a contribué, dans les années cinquante, à la formation du concept d'œuvre ouverte.

Berio est donc parti de l'organisation phonétique développée des poèmes pour structurer les paramètres les plus " anarchiques ", et leur donner une fonction structurante dans le discours musical. Son instrumentarium correspond déjà sur le plan acoustique à la distinction linguistique fondamentale entre consonnes et voyelles qui, dans le domaine musical, devient une opposition entre attaques et résonances. Chaque timbre instrumental peut, ensuite, s'appliquer aux différents types de consonnes et de voyelles, avec plus ou moins de précision, de sorte que le matériau englobe sons poétiques et sons musicaux dans une même unité.

Dans le premier mouvement de l'œuvre, par exemple, le /t/ de " stin- " génére l'accent vocal et l'attaque sèche de la harpe (unisson, accent, forte), suivis de mélismes et de résonances liés au phonème /i/. L'accent vocal souligne de la même façon les /g/ de "-ging " et " gold », repris par l'attaque de la harpe. 


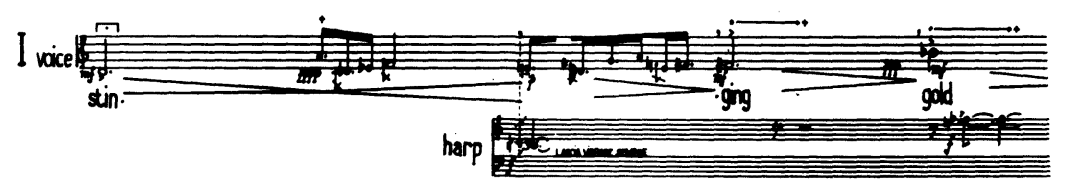

Copyright $\odot 1961$ by Universal Edition (London) Ltd., Londres.

Reproduit avec autorisation.

Berio: Circles (1960)

Exemple 1

D'autres phonèmes, dans ce premier poème, sont mis en évidence de cette façon. Mais Berio ne se contente pas d'une relation purement imitative : il fait proliférer le matériau musical. Ainsi, l'attaque initiale n'est pas un événement isolé, une qualité de jeu spécifique à ce moment de l'œuvre seulement, mais est développée en tant que catégorie compositionnelle importante pour la signification du mouvement. On remarque dans l'écriture de la harpe une grande variété d'attaques : unisson/corde simple, cluster/note isolée, forte/mezzo-forte/ piano, à la table/harmonique, percussion contre le bois de l'instrument, etc. La harpe nous conduit de la sonorité du /t/ énoncé par la voix au début jusqu'à l'entrée des instruments de percussion (temple block, wood block, log drum, claves ...) dans la coda (pp. 7-8 de la partition). Comme catégorie structurelle, l'attaque est articulée à l'écriture mélismatique d'une part, et au travail sur les résonances développé dans la coda à partir, là aussi, d'un élément phonétique. En effet, cummings sépare le " $S$ » de "dreams », le met en évidence, suggèrant une prolongation purement sonore du poème liée à sa signification :

$$
\begin{aligned}
& \text { " with } \\
& \text { dream } \\
& -S \text { " }
\end{aligned}
$$

Il y a transition de timbres entre ce " $-\mathrm{S}$ » de la chanteuse et le sand block, les maracas, le mexican bean, joués pianissimo, puis entre ces instruments joués crescendo et les claves, auquels se mêle la harpe. Les notes ponctuelles de la harpe engendrent à leur tour le temple block et le log drum. Les dernières mesures mélangent attaques sèches et trémolos soutenus, et aboutissent à l'entrée des percussions à hauteur déterminée (xylophone, marimba). 


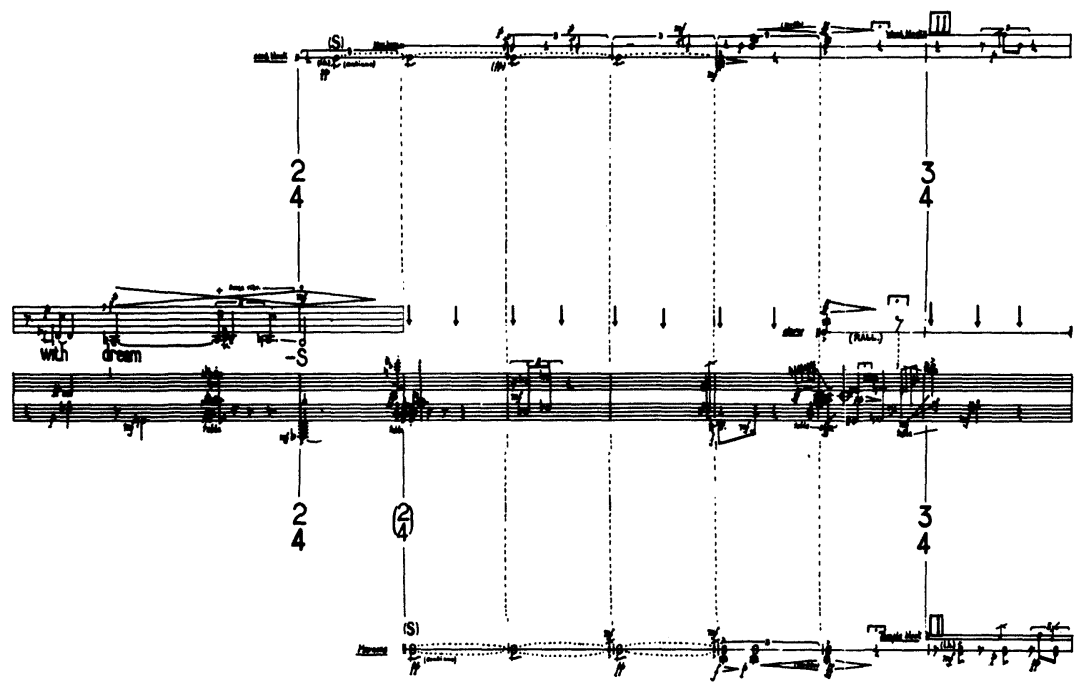

Copyright $\odot 1961$ by Universal Edition (London) Ltd., Londres. Reproduit avec autorisation.

Berio: Circles (1960)

Exemple 2

Dans le poème "riverly " (2ème partie), les trilles et les trémolos annoncés par la coda de "stinging " sont directement issus du phonème $/ R /$ ou / $/$ / qui joue un rôle essentiel (on le trouve au début et à la fin du texte, comme le /s/ dans « stinging " : " riverly " - "stare »; et il est dans le mot-clé « morte », au centre du poème. Or

... les deux espèces d'r se présentent comme un succession de petites voyelles séparées par autant de petites consonnes ... Les fluctuations de l'intensité le confirment, et les chutes consonantiques de la mélodie le corroborent à leur tour. On y reconnaît de véritables trilles ... (Morier 1961: 223).

Ces trilles et trémolos envahissent toute la texture de la seconde partie de Circles. Là encore, Berio part d'une caractéristique phonétique, et fait proliférer son matériau de façon autonome.

Les phonèmes générent aussi l'ordre d'apparition des timbres; à la fin du second poème, le mot "stare " provoque l'entrée des instruments métalliques, comme le $\mathrm{R}$ roulé avait auparavant permis l'entrée des instruments à peaux : 
... le /s/ engendre les cymbales suspendus (avec sizzles, ceci est important), lesquelles correspondent parfaitement aux exigences de durée, frottement et d'absence de son fondamental (suivent par sympathie le triangle et le tam-tam); le /t/ est prolongé par les cymbales à doigts, seulement au niveau de l'impact (explosion) et de l'absence de son fondamental . . .; la voyelle $/ \varepsilon /$ trouve sa contrepartie dans les hauteurs précises des timbales...; enfin le $/ \mathrm{r} / \mathrm{final}$, en tant qu'affaiblissement du $R$ roulé, permet le jeu, avec ou sans roulement, des peaux à hauteurs non déterminées (Demierre 1982: 51).

Si les éléments phonétiques des textes génèrent la construction du timbre et des intensités dans la partition, il semble que les paramètres de hauteurs et de durées soient rattachés au découpage syntaxique. Ils soulignent ou développent des articulations poétiques, les soumettant à leur propre organisation ou se soumettant à la leur. Berio utilise pour cela des figures stables, parfaitement identifiables, qui sont comme des objets musicaux autonomes se détachant du contexte. Ces figures à caractère gestuel, qui trouvent leur origine dans l'usage de la ponctuation ou des majuscules et minuscules chez cummings (voir « $\mathrm{n}(0) \mathrm{w} »$ ), fonctionnent comme des signaux, aussi bien sur le plan musical que sur celui des significations poétiques. Ainsi, dans "stinging", le point culminant du poème est souligné par deux figures gestuelles significatives qui sont réutilisées dans la coda (la première servant de " cadence " à la fin de cette coda).

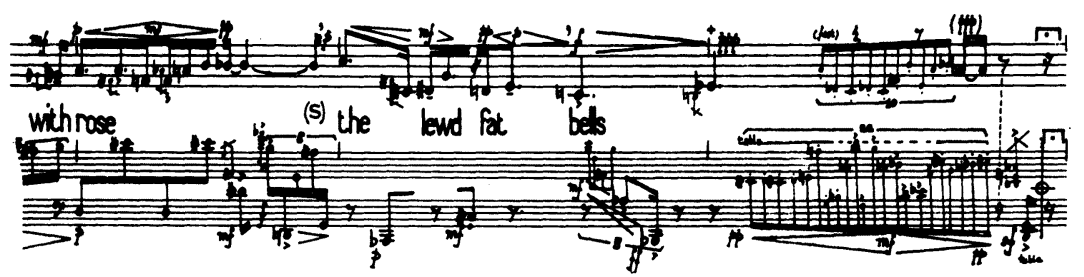

Copyright $\odot 1961$ by Universal Edition (London) Ltd., Londres.

Reproduit avec autorisation.

Berio: Circles (1960)

Exemple 3

On notera que ces deux figures amplifient de façon organique les deux caractéristiques d'écriture de cette première partie, telles que nous les avons relevées plus haut: un élément 
ponctuel sec (l'attaque) et un élément mélismatique. Par là, Berio crée une unité structurelle entre les différents paramètres, entre des éléments aux fonctions différentes.

Dans « $\mathrm{n}(\mathrm{o}) \mathrm{w}$ », Berio utilise une figure quasi-thématique qui permet de mettre en évidence le mouvement poétique, ses fausses répétitions, cette ambiguïté typique de cummings entre circularité et ouverture qui a fortement inspiré le compositeur : les mots « $\mathrm{n}(0) \mathrm{w}$ », «n,o; $\mathrm{w}$ : » et « N,ew » sont annoncés par le geste musical (voir les pp. 18, 22, 26 de la partition; cette figure apparaît pour la première fois dans la coda du deuxième poème, comme préfiguration purement musicale, à partir de laquelle cette figure est mémorisée et peut avoir une fonction de signal) :

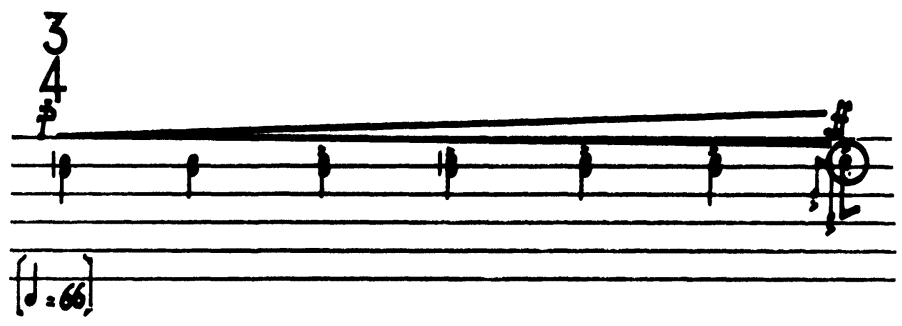

Copyright $\odot 1961$ by Universal Edition (London) Ltd., Londres. Reproduit avec autorisation.

Berio: Circles (1960)

Exemple 4

Les rapports entre structure poétique et structure musicale ne sont pas fixés, mais ils évoluent à travers l'œuvre. Berio a distribué les trois poèmes de cummings dans une forme en cinq parties :

\begin{tabular}{ccccc}
\hline I) & II) & III) & IV) & V) \\
stinging & riverly & $n(0) w$ & riverly & stinging
\end{tabular}

À l'évidence, il s'agit d'une construction en arche, où le poème « $\mathrm{n}(\mathrm{o}) \mathrm{w}$ » occupe une position centrale. Il est le point de fusion entre texte et musique. En effet, les phonèmes induisent ici les sons instrumentaux de façon généralisée : la musique absorbe le matériau poétique. Mais les hauteurs et les durées, relativement autonomes dans les deux premières parties, sont soumises à la présentation poétique : hauteurs approximatives, parlé, rythmes aléatoires. Le poème absorbe le matériau musical. Symboliquement, les instrumentistes mêlent phonème et son musical à la 
fin de cette troisième partie (pp. 27 et suivantes) et des réactions en chaîne s'instaurent entre la chanteuse et les instrumentistes (pp. 28 et suivantes).

Pourtant, cette symétrie formelle apparente est contrariée par un mouvement d'accumulation du matériau musical qui se manifeste à travers les fausses reprises que constituent les deux dernières parties de l'œuvre. Berio crée toutes sortes d'ambiguïtés : la reprise de "riverly " (IVème partie) s'approprie les hauteurs et l'unité de timbre (voix-harpe) de "stinging ", mais les durées diffèrent, et les accents ou les attaques qui étaient liés aux phonèmes de "stinging ", ont disparu au profit de la continuité qui caractérisait " riverly ". La reprise de " stinging " (Vème partie), elle, accumule le matériau de chaque partie de l'œuvre et amplifie, développe les attaques caractéristiques de la première partie, ainsi que le geste musical qui leur était lié (cf. plus haut). Dans ces deux dernières parties, Berio travaille à partir des unités musicales constituées. C'est un travail au second degré, la transformation non d'un matériau brut, ou du matériau poétique, mais de la musique composée dans les trois premières parties. Les fausses reprises condensent ce qui a été entendu, elles le transfigurent, nous invitant à une re-lecture de l'œuvre. Cette conjonction de la forme ouverte et de la forme circulaire est aussi, au moment où Berio compose Circles, une préoccupation de Boulez, et ce en rapport avec l'expérience des textes de Mallarmé : la 3ème Sonate et Pli selon Pli sont aussi organisés selon cinq mouvements "faussement " symétriques.

Pour le récepteur, la question qui est au centre d'une telle expérience, c'est la fonction de la répétition et le rôle joué par la mémoire. Berio (comme Boulez) évite toute répétition textuelle - la répétition en tant que réexposition. On ne trouve jamais les mêmes événements, ils sont filtrés par le temps de l'ouvre, transformés, déformés, chargés d'autres significations. La répétition apporte souvent une ambiguïté et non une réaffirmation. Elle ne rétablit pas une situation originelle mais en altère au contraire la perception et la signification. Elle agit rétrospectivement sur ce qui a été perçu, introduisant une distanciation, et faisant prendre conscience de l'écoulement du temps. C'est en ce sens-là aussi que l'oeuvre est ouverte : elle manifeste l'irréversibilité du temps, le caractère unique de chaque expérience d'écoute, une infinité d'approches possibles. 
Ce travail d'élaboration musicale à partir d'un texte, on le retrouve dans Sequenza $I I I^{7}$ pour voix seule, mais de manière assez différente. Berio utilise ici un poème moins élaboré que ceux de cummings, un poème " ouvert ", en raison de l'ambiguïté voulue des enchaînements syntaxiques, de leur permutabilité, et de l'incohérence sémantique qui en découle:

\begin{tabular}{|c|c|c|}
\hline $\begin{array}{l}\text { give me } \\
\text { to sing } \\
\text { to build a house }\end{array}$ & $\begin{array}{l}\text { a few words } \\
\text { a truth } \\
\text { without worrying }\end{array}$ & $\begin{array}{l}\text { for a woman } \\
\text { allowing us } \\
\text { before night comes }\end{array}$ \\
\hline
\end{tabular}

N'ayant pas à se soucier d'une forme poétique stricte, Berio fait éclater le poème de l'intérieur, transférant le « désordre " des enchaînements syntaxiques à l'enchaînement même des syllabes. Il poursuit là une démarche entreprise avec Visage (1961) et continuée avec $O$ King (1966) ou A-Ronne (1974-75) : l'utilisation de situations pré-linguistiques ou para-linguistiques, de construction du langage ou de quasi aphasie. Le texte de Kutter, réduit à ses unités minimales - unités dépourvues de sens -, est reconstruit par bribes, sans jamais retrouver sa forme initiale. Les éléments du texte sont traités comme un matériau sonore. Ils sont distribués selon deux techniques vocales principales : le quasi parlando (noté sur une seule ligne) et le chant (noté sur trois ou cinq lignes, selon que les hauteurs sont approximatives ou absolues), à quoi s'ajoutent des gestes vocaux (interjections, clicks, rires, bruits de souffle, etc.) ${ }^{8}$ Berio utilise toutes les ressources de la voix, de manière à englober musique, paroles, bruits, attitudes (ce qui élargit considérablement les catégories qu'avait relevé Stockhausen (1964) dans la partie chantée du Marteau sans maître de Boulez: parlando, quasi parlando, chant syllabique, chant mélismatique, chant bouche fermée). "Il n'est plus question désormais de texte à la musique, mais plutôt de qualités spécifiques de sons en différentes liaisons avec la signification $" .^{9}$

L'ensemble du matériau sonore apparaît très riche et relativement peu homogène si on se place d'un point de vue traditionnel; en essayant de le décrire, on remarque pourtant qu'il est sous-tendu par une organisation très hiérarchisée, le nombre fini d'événements sonores bien différenciés permettant de réaliser une transition sensible entre le bruit brut et la voix chantée. 


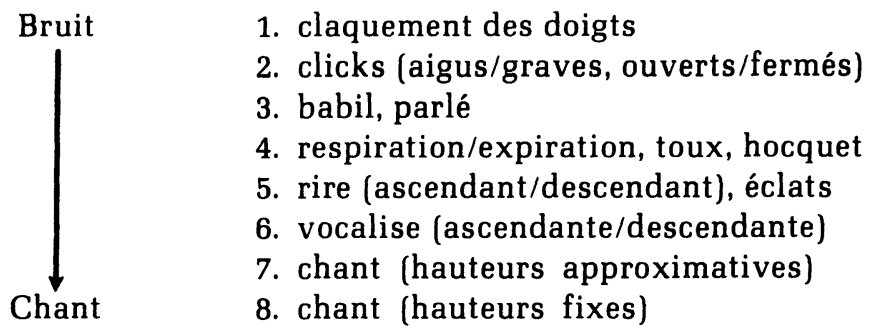

Du claquement de doigt au click, de la vocalise au rire, du parlando aux notes tenues avec changements de voyelles, du chant relatif au chant fixé, etc, on peut relever toutes sortes de transitions réalisées par simple glissement, par transformation progressive, ou par développement. Chaque catégorie paraît dominer une grande séquence : dans la première, c'est le parlando (jusqu'à $1^{\prime} 50^{\prime \prime}$ ); dans la deuxième, c'est le chant (jusqu'à $4^{\prime} 20^{\prime \prime}$ ); dans la troisième, c'est le rire et ses dérivés (cette séquence pourrait se subdiviser ainsi : rire-chant-rire) (jusqu'à $7^{\prime} 30^{\prime \prime}$ ); enfin le chant domine la séquence terminale. La trajectoire globale de la pièce montre la transformation d'un matériau purement phonétique (lié au babil dans les premières secondes) en la manifestation chantée, et le début de l'ouvre en donne une image condensée.

Si l'on prend n'importe quel passage dans la partition - par exemple ce début de l'ouvre - on est d'abord frappé par l'aspect discontinu et disparate des événements musicaux (cf. Ex. 5).

Les oppositions entre chaque segment ayant des particularités propres, des caractéristiques bien différenciées, donnent le sentiment d'une progression par ruptures. Par exemple, entre (al) et (bl), il y a opposition entre une figure amorphe (continuum dénué d'attaque, chute, accent, de mouvement de hauteur, de rythme et d'intensité) et une figure directionnelle (attaque nette, accents, mouvement rythmique et de hauteur). Mais si ces oppositions gouvernent l'enchaînement des segments au niveau du détail, le mouvement général réalise au contraire une transition repérable notamment par le biais des répétitions : le premier segment (al) se confond avec l'entrée de la chanteuse sur scène; il est donc mêlé aux applaudissements et doit s'arrêter avec eux. Il constitue la première transition entre le bruit de la salle et l'ouvre elle-même. Le second segment (a2) reprend la même figure en diminution, prolongée par la figure directionnelle de (b1). (a3) est à son tour prolongée par un click et une note chantée bouche 


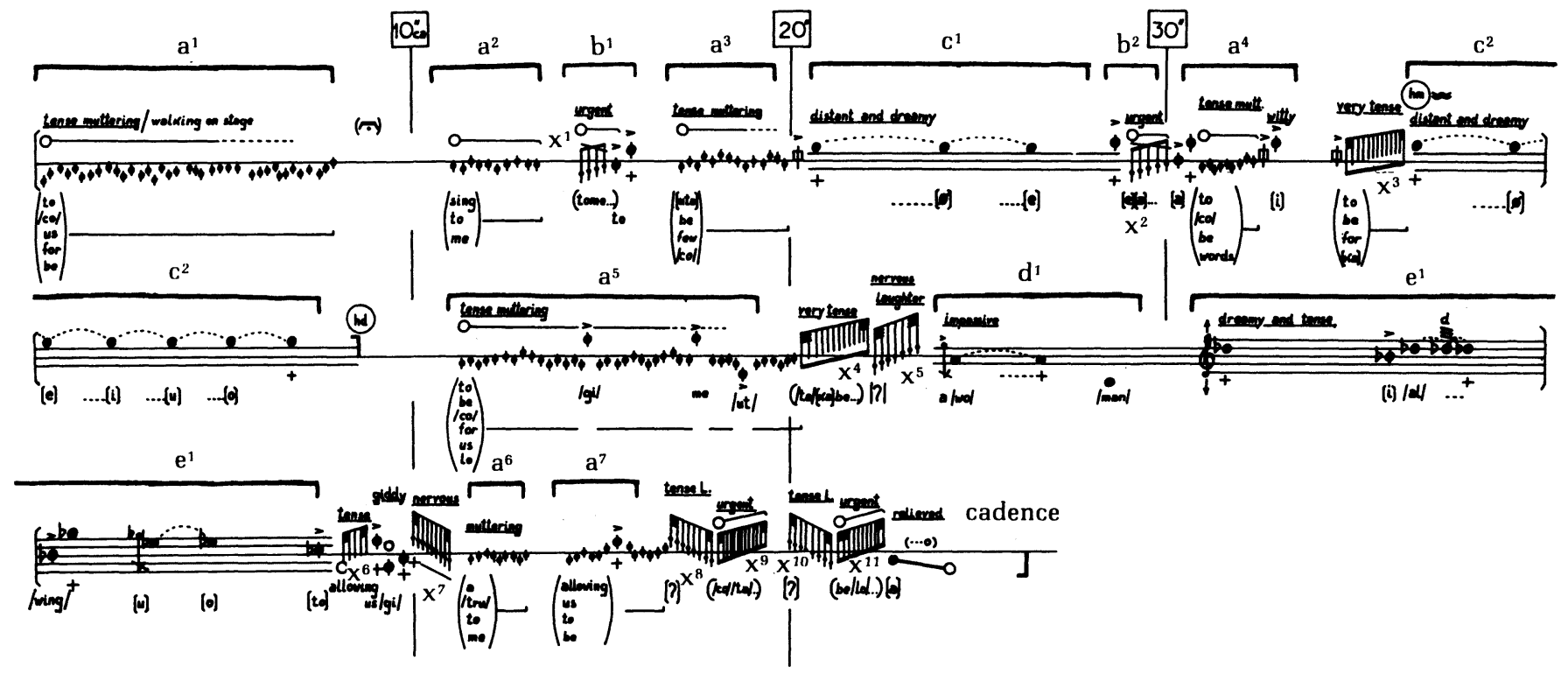

Copyright $\odot 1968$ by Universal Edition (London) Ltd., Londres.

Reproduit avec autorisation.

Berio: Sequenza III (1966)

\section{Exemple 5}


fermée (c1). Le chant est ainsi engendré à partir du bruit sans signification musicale qui précédait l'exécution. Mais il ne devient chant véritable que progressivement : d'abord bouche fermée, sur une note tenue, avec changements de timbre (c1), puis bouche ouverte, toujours sur une note tenue, la transformation continue du timbre par une succession de voyelles étant développée (c2); la figure ascendante rapide ( $x$ ) gagne de l'importance et introduit le chant noté sur trois lignes (d1). Le chant noté sur cinq lignes suit immédiatement (e1). Parallèlement, la figure rapide $(x)$ engendre un geste vocal essentiel dans Sequenza III : le rire. Lié aux figures musicales ascendantes rapides, puis plus tard aux vocalises, il est pris dans une chaîne de transformations purement musicales, mais il demeure reconnaissable en tant que rire, et par là, échappe à la hiérarchie purement musicale : c'est une figure ambiguë.

Chaque élément est, de la sorte, lié à des figures qui le préparent et qui le développent, qui peuvent appartenir à la fois à l'organisation musicale et à un réseau de connotations extramusicales. La mise en parallèle des figures appartenant à une même famille $(a 1, a 2, a 3 \ldots / b 1, b 2 \ldots . /$ etc $)$ montre clairement l'importance des répétitions structurelles (et non pas textuelles) auxquelles Berio est toujours resté très attaché.

En même temps, on peut dégager des couples d'oppositions selon les caractéristiques de chaque famille d'éléments :

\footnotetext{
hauteurs pertinentes / hauteurs non pertinentes son pur / son complexe continuum / événement ponctuel

figure amphore / figure directionnelle etc.
}

On retrouve ces oppositions à un niveau plus général, entre voix chantée et voix parlée, entre sons articulés et babil, entre structure musicale et geste vocal, etc. (nous avons donné un exemple supra, entre a2 et b1).

La sélection d'un matériau qui répond à ces deux types d'organisation - contiguïté (horizontalité) et opposition (verticalité) - permet une véritable dialectique compositionnelle. Les principes d'oppositions et de transitions deviennent tout à fait opératoires. Chaque événement musical est ainsi chargé de sens multiple, à l'image du rire, qui est à la fois caractéristique en tant qu'événement individuel (donc parfaitement repérable à l'audi- 
tion) et interchangeable en tant que structure musicale. Berio développe en cela le principe du poème de Kutter pris pour base de Sequenza III : les phonèmes, les mots, les phrases y sont permutables, selon qu'on s'attache aux différents sens possibles ou à la construction phonétique.

Evidemment, cela ne se fait pas mécaniquement : certaines caractéristiques filtrent à l'intérieur de figures qui les excluaient à l'origine. La composition met en relation perpétuellement renouvelées les différents éléments de l'œuvre, en élimine certains définitivement, et déjoue ainsi tout schématisme. La capacité que possède le processus compositionnel de sans cesse remettre en question les hiérarchies qu'il a lui-même instaurées, est l'un de ses aspects les plus fascinants (et l'un des plus importants dans toute l'histoire musicale). L'œuvre, tel un organisme vivant, échappe au schéma figé de la forme, en fonction de sa logique propre, mais aussi de la manière dont l'auditeur la saisit. Elle se refuse à toute totalisation rationnelle.

Dans le matériau que nous avons analysé, il est un élément de première importance : les indications de jeu, qui sont au nombre de ... quarante-quatre! Elles constituent un véritable répertoire d'émotions ou d'attitudes : «inquiet », " calme », " rêveur », " joyeux », " désorienté », « timide ", " langoureux ", " serein », " plaintif », « pressant », « tendu », etc. Pourtant, elles n'ont pas de valeur psychologique ou de fonction réaliste, mais sont des paradigmes d'attitudes et d'émotions. Sequenza III qui a toutes les caractéristiques d'un monologue - Cathy Berberian a même usé du terme "radiographie de l'âme féminine " -, n'a aucunement une dimension narrative ou anecdotique. L'excès d'indications expressives en est d'ailleurs l'indice; Berio prévient la chanteuse qu'elle « ne doit pas essayer de représenter ou de mimer la tension, la pression, la distance ou le rêve ... » (note de la partition). Le compositeur traite ces indications comme il traite les gestes vocaux ou les bruits, comme un matériau n'ayant pas seulement une valeur en-soi, mais pouvant entrer dans un système de relations qui devient à son tour signifiant. Dans un passage comme l'exemple suivant, basé sur le rire, les oppositions radicales et extrêmement rapides d'expressions empêchent une saisie au premier degré : le processus de condensation devient par lui-même signifiant (cf. Ex. 6 ci-contre).

Ces indications de jeu, comme les modifications d'émissions vocales, sont en général liées aux figures musicales, mais elles acquièrent aussi par instant une certaine indépendance; elles 


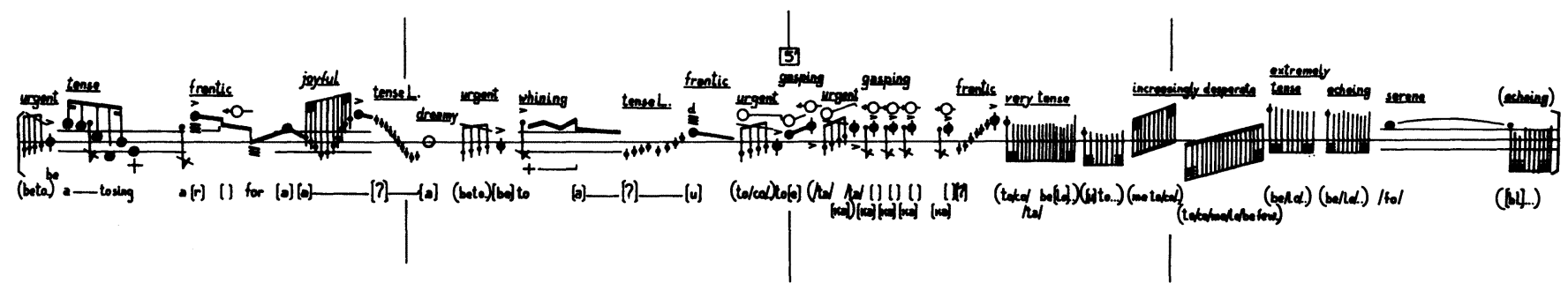

Copyright $\odot 1968$ by Universal Edition (London) Ltd., Londres.

Reproduit avec autorisation.

Berio: Sequenza III (1966)

Exemple 6 
peuvent jouer le rôle principal dans la caractérisation et le sens d'un passage, au même titre que les gestes vocaux ou le chant proprement dit. La partition révèle ainsi une polyphonie qui est moins une polyphonie de notes qu'une polyphonie d'actions (on retrouve un tel geste chez Berio dans d'autres oeuvres, telles Sequenza $V$ pour trombone, très proche de la III, ou dans Sinfonia, Laborintus, A-Ronne, etc.). Ainsi dans l'exemple suivant, on distingue trois niveaux : la note chantée, avec des accents; les modifications de timbre dues aux éléments phonétiques (a), (i), (e), /to/, /be/; et les indications de jeu, "dreamy ", "intense ", " dreamy », « intense », " distant ».

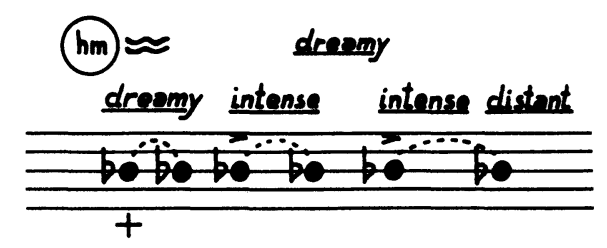

[a] [e] [i] to be

Copyright $\odot 1968$ by Universal Edition (London) Ltd., Londres.

Reproduit avec autorisation.

Berio: Sequenza III (1966)

Exemple 7

On pourrait dire que dans Sequenza III, comme dans la plupart des Sequenza d'ailleurs, il y a une polyphonie latente, qui devient manifeste à certains endroits. On peut considérer également la succession d'événements sonores hétérogènes et les répétitions ou transformations à distance comme le déploiement monodique d'une polyphonie virtuelle.

On peut noter que dans Sequenza III, il n'y a aucune indication d'intensité (il faut se reporter aux indication "expressives "), ni de rythme (des repères en secondes déterminent des champs de durées à l'intérieur desquels la chanteuse répartit les figures musicales). Une écriture rythmique précise, difficilement réalisable avec certains éléments sonores utilisés, eût globalisé l'ensemble des événements en les rapportant à une pulsation, à un tempo. Au contraire, la construction et la perception du temps dépendent de la densité de chaque événement et de ses relations aux autres, et non d'un étalon fixe, pré-établi : les séquences homogènes, par exemples celles où le chant domine, 
seront perçues très différemment de celles qui, comme dans l'exemple 6, présentent des ruptures et une condensation d'éléments très différents. La capacité de la chanteuse à maîtriser ces brusques changements joue évidemment un rôle dans l'effet produit. Le temps musical est donc lié aux tendances du matériau et aux critères d'exécution, non à une forme définie globalement. L'auditeur n'a plus de repères " abstraits » (division de la forme fonctionnelle, comme dans le rondo ou la formesonate), la perception de l'événement isolé ne dépend plus pour lui d'une conscience globale de l'ouvre. Au contraire; il doit partir du détail, et dans l'accumulation imprévisible des séquences, (re)construire le temps de l'ouvre. La mémoire ne peut plus fonctionner comme un moyen de " conceptualiser " le discours musical, mais elle enregistre chaque événement en essayant de le rapporter plus ou moins librement au stock déjà accumulé et disponible. D'où les idées d'ouverture et d'un temps musical relatif.

L'œuvre ouverte rompt avec l'idée d'un espace absolu et d'un temps linéaire, elle rompt avec l'idée même d'un centre. Elle tend au contraire à accumuler des instants, des morceaux de présent presque autonomes, qui provoquent une fragmentation à la surface de l'ouvre. ${ }^{10}$ De cette fragmentation naît la possibilité d'un jeu infini d'analogies à distance, de rapports fondés non plus sur l'identité interchangeable entre les éléments, mais sur la convergence des fonctions structurelles. Les divers paramètres, par exemple, peuvent être à tour de rôle pertinents pour le sens de l'ouvre : il n'y a plus une hiérarchie fixée (la hauteur dominant le timbre, par exemple) mais une hiérarchie mobile qui est modifiée à l'intérieur de l'oeuvre. C'est en définitive le matériau qui détermine, pour chaque ouvre, la "stratégie " compositionnelle nécessaire. L'ensemble du matériau, dans Sequenza III, renvoie aux conventions liées à la voix et au corps, à des usages socialement, culturellement et historiquement différents. L'expérience du matériau est, pour Berio, confrontation avec la réalité et avec l'histoire. D'où l'importance de l'élément gestuel et d'une théâtralisation latente.

Le paramètre des hauteurs, dans des oeuvres comme Circles ou Sequenza III, a souvent une fonction secondaire, il est articulé à d'autres modes de structuration, liés au timbre ou aux intensités par exemple. Il est néanmoins soigneusement organisé, et non laissé au hasard. Dans ces deux ouvres, les rapports de secondes mineures/majeures et de tierces mineures/majeures 
dominent largement. Dans Sequenza III, les parties chantées (notées sur cinq lignes) laissent même apparaître des structures harmoniques sous-jacentes, et une polarisation sur l'accord de sol bémol : on trouve au début la succession réb-sib-solb, au centre exact de la pièce $\left(4^{\prime} 20^{\prime \prime}\right)$, sur le mot " truth ", la note fa dièze, tenue jusqu'au chuchotement, et à la fin, l'insistance sur la tierce sib-solb par laquelle l'œuvre se termine. Des répétitions variées, jamais textuelles, assurent tout au long de la partie chantée, une certaine cohérence. A ce matériel réduit, sur le plan de l'organisation des hauteurs, correspond une différenciation maximale dans le timbre, les modes de jeu ou l'expression.

La formation classique requise pour $O K$ King, ${ }^{11}$ c'est-à-dire la formation du Pierrot lunaire de Schoenberg, privilégie le paramètre des hauteurs que Berio dote d'une fonction principale. Dans Circles et Sequenza III, la dimension gestuelle avait un sens à la fois musical et réaliste; elle s'appuyait sur un matériau hétérogène. Dans $O \mathrm{King}$, Berio ne renonce pas à cette dimension, mais elle s'applique à un matériau plus homogène. L'organisation des hauteurs demande, pour cela, d'être analysée de façon détaillée. ${ }^{12}$

Le texte, dans cette ouvre, est réduit à la simple énonciation du nom du pasteur noir américain, assassiné en 1968 : «O Martin Luther King! ». Mais la chanteuse n'utilise, pendant les deux tiers de la pièce, que les phonèmes constitutifs de cette phrase. Ils génèrent une part du matériau sonore, par l'opposition entre les voyelles - /o/, /a/, /i/, /u/, /3/, / / - et la consonne occlusive $/ \mathrm{k} /$, opposition traduite sur le plan des intensités par le rapport entre une couche sonore notée $p p p p$, faite d'un continuum où les attaques sont imperceptibles, et des accents brefs, incisifs, notés $f f$, qui le déchirent. On a donc une structure linéaire fondée sur la chaîne des voyelles, qui offre une transition continue du /a/ au $/ \eta /$, et une structure ponctuelle, violente, liée à la consonne $/ \mathrm{k} /$. Les instruments utilisent différentes techniques de jeu pour réaliser des changements de timbre progressifs (jeu sul ponticello, con sordino, vibrato et non vibrato, harmoniques, trémolos, etc.). Mais il s'agit là des caractéristiques les plus apparentes. Nous avons dit que le paramètre des hauteurs était prédominant; il est organisé sériellement et construit selon deux structures spécifiques : 
la première est formée de sept hauteurs gelées -, c'est-à-dire fixées une fois pour toutes quant à leur tessiture - organisées mélodiquement en un refrain de vingt et une notes,

la deuxième structure, jouée presque uniquement par le piano, est plus ornementale; elle complète l'échelle chromatique en utilisant les cinq hauteurs restantes, mais permute petit à petit leur tessiture. Si la première structure est écrite dans une tessiture moyenne, la seconde, plus étendue, l'enveloppe:
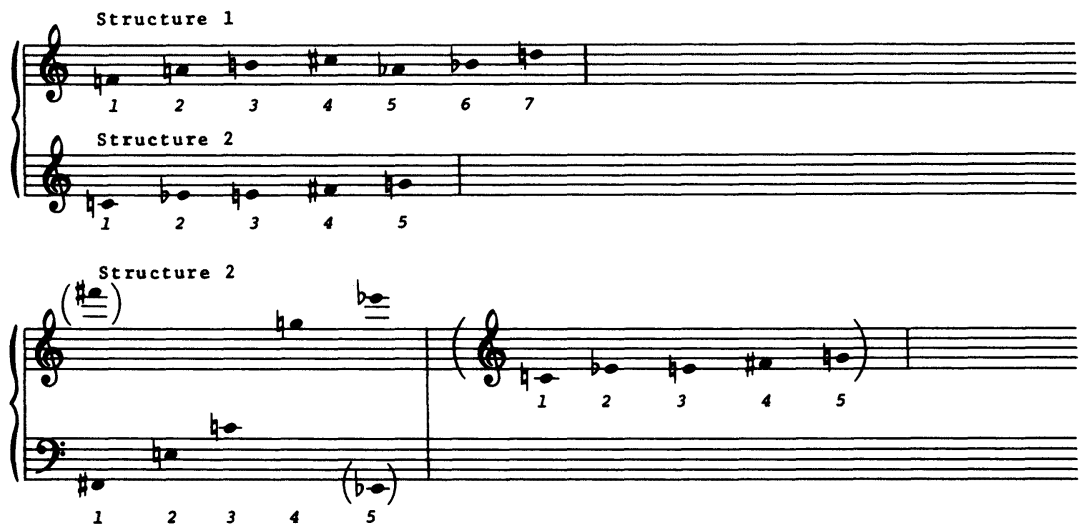

Exemple 8

La répétition joue un rôle important dans la première structure : la construction du refrain se fait en spirale, par ajouts successifs autour des notes-pivot. Cela peut rappeler certaines constructions de chansons issues du répertoire oral. Les trois segments (a-b-c) croissent de façon régulière par un ajout de trois notes et sont organisés dans un rapport de gamme par tons entiers :

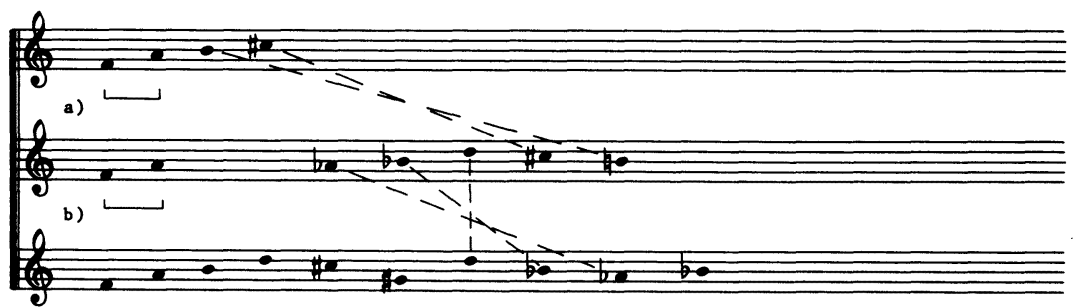

c) ᄂ

Exemple 9 
Berio joue des permutations, de l'introduction d'une note ou d'un groupe de notes dans une structure donnée, à la manière de tropes miniatures; le rapport fa-la reste inchangé, c'est lui qui donne le signal des différents segments. Le retour périodique sur cet intervalle, prolongé dans les parties instrumentales, donne l'illusion d'une polyphonie virtuelle, comme si l'on avait des entrées successives de voix différentes. Cette construction offre en miniature l'image de la construction formelle, chaque refrain (indiqué dans la partition par une lettre) déroulant les vingt et une notes dans le même ordre avec des variations de rythme et de densité harmonique à partir de cet intervalle fa-la.

Les instrumentistes modifient insensiblement l'arrière-plan harmonique en utilisant exclusivement les sept notes énoncées selon toutes sortes de combinaisons. Il ne s'agit pas d'une mélodie accompagnée, au sens traditionnel, mais de la fusion entre la dimension mélodique et la dimension harmonique (la voix doit se fondre dans le timbre instrumental, selon une note de Berio dans la partition). A l'intérieur de ce continuum défini par un choix de hauteurs restreintes et d'une tessiture limitée, les accents dessinent à leur tour, de façon anagrammatique, les vingt et une notes du refrain. C'est une sorte de cantus firmus caché. A la neuvième mesure de $\mathrm{D}$, les accents ayant épuisé la série de vingt et une notes, les quatre premières sont reprises de façon resserrée, presque dramatique :
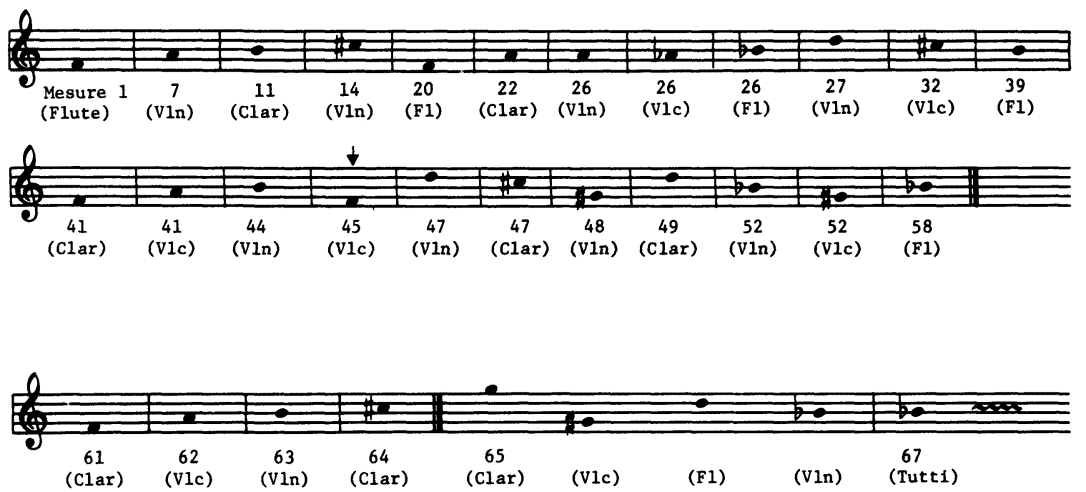

Exemple 10 
Tandis que les instruments se fixent progressivement sur le sol (note la plus aigue du refrain), la voix articule de plus en plus clairement le nom de Martin Luther King. A partir de D, on peut lire : «o-(3)(o)ma-(i)-lu (i) lu-(i)-(3) (o)-ma-(i) lu (3) King». C'est sur ce mot, deux mesures avant $\mathrm{E}$, que les musiciens retrouvent l'unisson initial, mais cette fois sur la note sib. Parallèlement, la deuxième structure a permuté progressivement toutes ses notes en conservant les rapports de tessiture initiaux.

La coda offre alors un renversement des données : tandis que la voix, qui s'individualise, formule enfin le nom du pasteur assassiné, les instrumentistes brouillent les structures instaurées : six accords composés des mêmes notes mais permutées reprennent les cinq hauteurs de la deuxième structure; le piano joue des clusters et une succession de deux fois trois notes, dans le registre grave, basées sur des rapports de tons entiers et comportant des octaves (cf. Ex. 11).

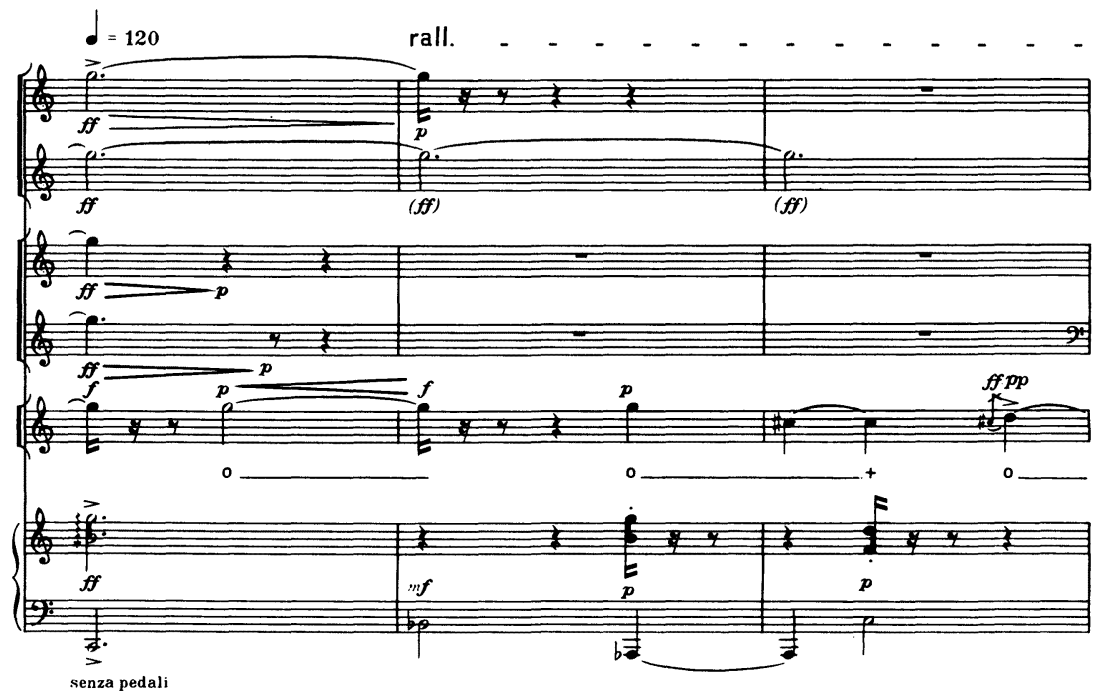



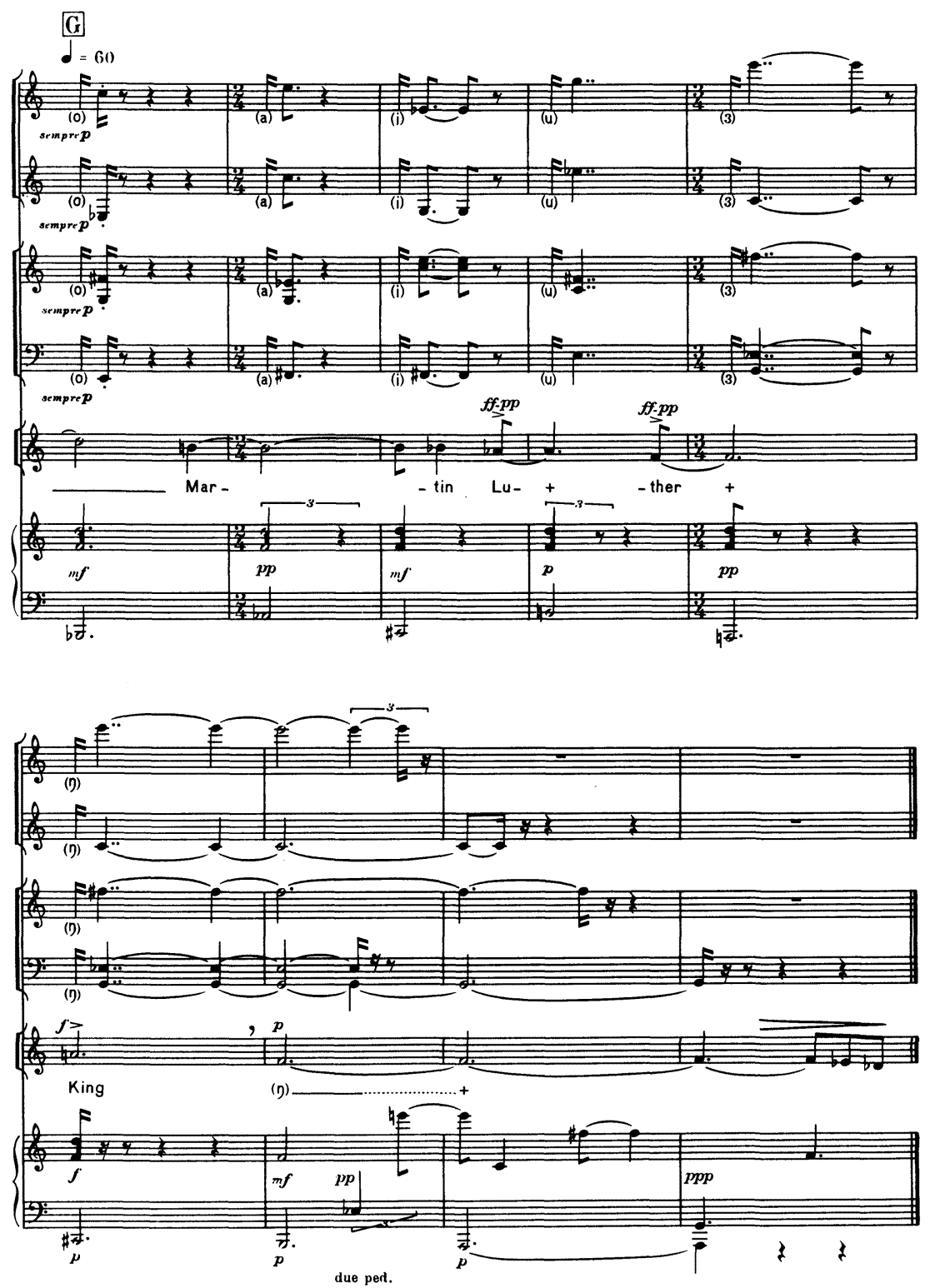

Copyright $\odot 1970$ by Universal Edition (London) Ltd., Londres.

Reproduit avec autorisation.

Berio: O King (1968)

Exemple 11 
Ce croisement des structures trouve un équivalent sur le plan du matériau phonétique, puisque derrière le nom de Martin Luther King énoncé par la voix, les instrumentistes reprennent la série des voyelles, leur conférant, juste avant chaque accord, une fonction d'attaques liée auparavant à la consonne /k/. Berio s'attache donc ici au processus de formation du sens à partir d'un matériau sonore non signifiant. Dans une première partie, ce matériau est utilisé dans une structure musicale rigide, parfaitement organisée; dans la coda, cette structure musicale est brouillée, et c'est alors le sens du texte qui apparaît. Dans Sinfonia (1968-69), $O$ King a été repris comme second mouvement (Berio a conservé la même structure mais distribué le matériel pour huit voix et orchestre). Il apparaît encore au cinquième mouvement (mouvement rajouté par la suite qui reprend des fragments des mouvement précédents et se conclut symétriquement au premier mouvement) où le refrain est progressivement recouvert par un roulement de caisses claires. $O$ King, rigoureusement construit sur le plan des hauteurs, est englouti, submergé par le bruit. La symbolique que l'ouvre dévoile par sa construction même, prend ici une nouvelle dimension dans ce moment de réapparition-disparition.

J'ai essayé de montrer comment la composition pouvait, de manières très différentes, organiser un matériau donné. Ce matériau, chez Berio, est souvent hétérogène: le musical, aujourd'hui, n'est plus limité aux notes de la gamme, à ce qui est filtré par elles. En conséquence, la composition ne peut guère espérer retrouver des critères absolus, fixés dans un espace bien délimité. A l'encontre d'une mode qui traque la tonalité dans les ouvres atonales et rêve d'un nouveau langage universel, je crois que l'extension du matériau musical durant ce siècle interdit une telle réification. La composition, qui s'est libérée des schémas pré-conçus, des systèmes généralisants, doit au contraire sans cesse réinventer sa liberté si elle veut nous faire entendre l'inouï et percer les apparences. Les oeuvres de Berio peuvent servir d'exemples pour une telle exigence.

J'ai insisté sur la cohérence de l'œuvre. Ce n'est pas que Berio néglige les connotations réalistes du matériau qu'il utilise, ni la possibilité de produire, à l'aide de structures musicales, une multiplicité de significations et d'émotions, ou une sorte de 
théâtralité latente également présente dans beaucoup de ses oeuvres instrumentales. Les sons ou les structures sonores ne sont jamais, chez lui, isolés dans un système formalisant qui se suffirait à lui-même. Ils sont toujours expressifs, mais dans un sens qui dépasse la seule problématique d'un Sujet individuel : ils expriment le réel et le collectif (sous ses formes mythiques, politiques, quotidiennes ...) dans toute leur complexité. Les actions musicales dans Circles, les gestes vocaux dans Sequenza III, les structures harmonico-mélodiques dans $O$ King peuvent être pris comme autant de symboles qui renvoient à la réalité vécue par chacun et lui donnent une dimension plus profonde, plus générale, presque emblématique. La composition, qui s'approprie un matériau très divers, devient alors productrice de significations multiples et non seulement musicales, et ce de façon très concrète, dans son processus même, dans le mouvement qui la manifeste.

L'oeuvre, en effet, ne trouve sa cohérence qu'en elle-même, et non dans le flot d'associations ou de significations diverses qu'elle entraîne. Il me semble que deux critères s'imposent pour cette unité musicale : un nombre d'événements limités, assimilables par la mémoire, offrant des caractéristiques d'opposition et de transition entre eux; et la possibilité de leur donner une fonction à travers le processus de composition (hiérarchie dans la présentation, répétitions, transformations, combinatoire, etc), c'est-à-dire une dimension structurelle effective. Le matériau utilisé peut, à ces conditions, être l'ensemble des notes tempérées, ou un ensemble de "bruits " choisis, la sélection opérant, dans tous les cas, un rôle essentiel qui est à la base même du travail de composition.

Berio a, dans la plupart de ses oeuvres (et plus que jamais dans celles de ces dix dernières années) renoué avec le principe de la répétition, sur lequel pesait l'interdit des compositeurs sériels dans les années cinquante. Par là, il a réactivé l'idée thématique ou motivique qui en découle, mais de façon élargie.

Ainsi de la structure de refrain dans $O$ King, ou d'un élément gestuel comme le rire dans Sequenza III, ou encore de certains gestes instrumentaux dans Circles. Le caractère " thématique " est lié chez le compositeur à l'idée de geste musical, figure ambiguë relevant à la fois du sens proprement musical et de significations associatives. De même, Berio définit dans la plupart de ses oeuvres, une sorte de champ harmonique (le mot " harmonique » étant pris dans un sens large) propre à chaque 
pièce : dans $O$ King, il est traditionnellement défini par la structure de tons entiers et par l'usage de tessitures absolues; dans Circles ou Sequenza III, il inclut les éléments phonétiques et les sons qui en dérivent, le répertoire des indications de jeu, l'ensemble des gestes vocaux. Contrairement à l'harmonie tonale qui a une fonction à la fois architectonique et dynamique, les champs harmoniques chez Berio ont un rôle statique : ils définissent un certain espace musical à l'intérieur duquel les éléments " individuels » sont constamment permutés et modifiés, créant des relations toujours renouvelées entre eux. De là peut-être la possibilité pour Berio de développer des techniques répétitives (comme dans Coro ou dans Sequenza VIII pour violon), et d'intégrer la structure modale des musiques populaires dans certaines de ses oeuvres (les meilleurs exemples d'une telle intégration se trouvent dans les dernières oeuvres : Coro, 197476 et La Vera Storia, 1977-78).

Si les ouvres de Berio transforment en musical ce qui ne l'est pas, et ouvrent le musical à d'autres modes de significations, de relations, ou d'émotions, c'est à chaque auditeur d'établir ces connexions qui n'apparaissent jamais comme des tautologies, qui n'ont pas de caractère systématique.

La musique devient alors, comme dit Berio,

un instrument intellectuel comme un autre, un instrument de connaissance de soi et de transformation, permettant à l'homme d'établir des rapports entre les choses et d'affronter ce dilemme ancien (cette oeuvre éternelle qui ne cesse de se poursuivre, devrait-on dire) : réaliser une unité, une belle harmonie, entre l'intellect et les sens, ou, comme l'aurait dit nos ancêtres, entre l'« âme » et le « corps ». 


\section{ANNEXE: Textes de Circles}

\section{IMPRESSION (V)}

Stinging

gold swarms

upon the spires

silver

chants the litanies the

great bells are ringing with rose the lewd fat bells and a tall

wind

is dragging

the sea

with

dream

$-\mathrm{S}$

\section{POST IMPRESSION A}

Riverly is a flower gone softly by tomb rosily gods whiten befall saith rain

anguish

of dream-send is hushed

in

moan-loll where

night gathers

morte carved smiles

cloud-gloss is at moon-cease soon

verbal mist-flowers close

ghosts on prowl gorge

sly slim gods stare
Saississants

ors essaimant

sur les spires

argents

chantant les litanies les

grosses cloches sonnent le vermeil les lascives grasses cloches et un haut

vent

drague

la mer

avec

ses rêve

$-S$
Riverainement est une fleur allée doucement à tombe roségaiement des dieux blanchissent adviens dict la pluie angoisse une dhoulde fin-de-rêve est impétue

en

gémis-situde où

la nuit cueille

des ris sculptés de mort

brillant-de-buée est à lune -arrêt bientôt

de verbales fleurs-de-brume se closent

des spectres en rôde se gorgent

de madrés menus dieux fixi-regardent 


\section{NOTES}

1. Programme des représentations d'Opera à l'opéra de Lyon, saison 1979-80.

2. Cf. I Novissimi (manifeste, anthologie), paru à Milan en 1961 et Gruppo 63, critica e teoria rassemblés en 1976 par R. Barilli et A. Guglielmi. Sanguinetti, par son recueil de poèmes Laborintus (1956) est à l'origine du mouvement avant-gardiste italien. Laborintus est reproduit in Contrechamps, $\mathrm{N}^{\mathrm{o}} 1,1983,75-82$.

3. Interview de Luciano Berio par Maurice Fleuret, Nouvel Observateur, $\mathrm{N}^{\circ} 153$, octobre 1967, p. 52.

4. Interview de Cathy Berberian, Des femmes en mouvement, mars 1979 et octobre 1981.

5. Interview de Luciano Berio par Martine Cadieu, Lettres françaises du 6 décembre 1967.

6. Circles, 1960; Universal Editions No 13231, 1961. Je dois au remarquable travail de licence de Jacques Demierre : Circles, ee cummings lu par Luciano Berio, Université de Genève, l'essentiel de mon information au niveau de l'analyse. Ce travail est repris en partie in Contrechamps, N"1, 1983, 123-180.

7. Sequenza III, 1966, Universal Editions No 13723, 1968. Une analyse de l'œuvre, du point de vue du rôle des affects, de la transgression des codes, etc., a été publiée par Lyotard et Avron (1971). Cf. aussi, pour une préoccupation assez proche, l'étude de Anhalt (1973).

8. Jakobson, citant Van Ginneken, parle de geste vocal à propos du click : " Les gestes vocaux en général et les clicks en particulier forment une couche prélinguistique, extralinguistique, et l'on pourrait ajouter post-linguistique, comme nous le montre l'étude de l'aphasie " (1969: 100).

9. Interview par Martine Cadieu citée.

10. Cf. aussi la théorie de la Moment-Form chez Stockhausen (1964). Dans un article intitulé "Invention et découverte", il écrit : "Composer des états et processus à l'intérieur desquels chaque moment constitue une entité personnelle, centrée sur elle-même et pouvant se maintenir par elle-même, mais qui se réfère, en tant que particularité, toujours aussi à son contexte et à la totalité de l'oeuvre».

11. O King, 1968; Universal Editions, No 13781, 1970.

12. L'analyse de Stoïanowa (1978: 168 et sq.) sur $O$ King nous semble, de ce point de vue, rester à mi-chemin dans l'étude de la structure de l'œuvre. 
ADORNO, T. W.

\section{RÉFÉRENCES}

1982 : Quasi una fantasia : vers une musique informelle. Paris : Gallimard.

ANHALT, I.

1973 : «Luciano Berio's Sequenza III », Les Cahiers canadiens de musique, $\mathrm{N}^{\circ} 7,23-60$.

BERIO, L.

1959 : "Poesia e nuusica - un'esperienza », Incontri musicali, $\mathrm{N}^{\circ} 3$, 98-111. Trad. française in Contrechamps, $N^{\prime \prime} 1,1983,24-35$.

1966 : "Façon de parler ", Preuves, N"180, 30-31.

BORNOFF, J.

1973 : "Music, Musicians, and Communication : Five Interviews (1. Luciano Berio) ", Cultures, I/1, 113-122.

BOULEZ, P.

1963 : Penser la musique aujourd'hui. Paris : Gonthier.

1964 : "Sonate, que me veux-tu? », Médiations, $N^{\circ}$ 7, 61-75; repris in Points de repère. Paris : Bourgois, 1981, 151-163.

DEMIERRE, J.

1982 : Circles : e. e. cummings lu par Luciano Berio, mémoire de licence, Université de Genève.

ECO, U.

1962 : Opera Aperta. Milan : Bompiani; trad. fr. L'œuvre ouverte. Paris : Seuil, 1965.

JAKOBSON, R.

1969 : Langage enfantin et aphasie. Paris : Minuit.

KAGEL, M.

1966 : « $1965=1937$ », Preuves, $\mathrm{N}^{\circ} 181,42-45$.

LYOTARD, J.-F. et AVRON, D.

1971 : "A few words to sing - Sequenza III », Musique en jeu, $\mathrm{N}^{\mathrm{o}} 2,30-44$.

MORIER, $\mathrm{H}$.

1961 : Dictionnaire de poétique et de rhétorique. Paris : Presses Universitaires de France.

STOCKHAUSEN, $\mathrm{K}$.

1964 : "Musik und Sprache I ", in Texte, Vol. II. Cologne : Dumont Schauberg, 149-156.

STOÏANOWA, I.

1978 : Geste - Texte - Musique. Paris : Union générale d'éditions (10-18). 